\title{
Partially resolved super transition array method
}

\author{
Brian G. Wilson, Carlos A. Iglesias And MaU H. Chen \\ Lawrence Livermore National Laboratories \\ P.O. Box 808, Livermore, CA 94550, USA
}

\begin{abstract}
The partially resolved transition array model for radiative transitions between ordinary electronic configurations is extended to the super configuration approach. The specific application incorporates intermediate coupling effects into transition arrays to describe the transition from LS to JJ coupling without the ad hoc approximations introduced in past formulations. More generally, the extended concept permits stepwise refinement of the super transition array method towards the line-by-line limit in ordinary configurations. Thus, the formalism forms a framework for a hybrid scheme to combine detailed line accounting and statistical methods.
\end{abstract}

Keywords:

Plasma spectroscopy, transition arrays, opacity, emissivity, configuration interaction, intermediate coupling

Corresponding author:

e-mail: wilson9@1lnl.gov (B.G. Wilson) 


\section{Introduction}

For plasmas containing partially ionized atoms the detailed line accounting (DLA) method is, in principle, the ideal for calculating bound-bound spectra. For complex ions, however, the myriad spectral lines make DLA calculations impractical [1], even under the common approximation of neglecting configuration interactions (CI). To circumvent this impasse, calculations resort to statistical treatments, namely the unresolved transition array (UTA) approximation, where all spectral lines from a configuration-to-configuration transition array are treated as a single spectral feature [2,3]. The UTA model gives compact formulas for the strength-weighted energy mean and variance of the array, which together with a Gaussian approximation affords a fast spectrum calculation.

For very large numbers of configurations even the UTA approximations become unfeasible. In this case the super transition array (STA) method [4,5] has proven a powerful technique to model plasmas. The STA method can account for all possible bound-bound radiative transitions in the plasma by collecting ordinary configurations into a single entity, the super configuration, significantly reducing the computational effort. The STA method replaces many UTA's by a single Gaussian profile with strength-weighted moments obtained from compact formulas; thus, avoiding the explicit summation over the individual UTA's.

Along with its computational advantages the STA approach, however, carries certain defects such as inaccurate higher moments or excessive merging of spectral features. Furthermore, no exact statistical formalism exists to describe the evolution from LS to JJ coupling. That is, statistical descriptions of transition arrays with the UTA or STA approximations rely on pure LS or JJ angular momentum coupling. The LS coupling is valid when spin-orbit terms are weak compared to the electrostatic interactions. The transition array is then described by a single Gaussian characterized by the line strength, energy centroid, and variance obtained from the LSUTA formulas [2]. These moments can be made near exact if the spin-orbit parameter is computed non-perturbatively. For cases with significant spin-orbit interaction, however, the single Gaussian feature is too crude.

In the opposite limit when spin-orbit interactions are much stronger than the Coulomb terms, $\mathrm{JJ}$ coupling is appropriate. The spectrum is now split into 2 or 3 distinct spin-orbit split arrays (SOSA) each with its own strength, centroid, and variance [3]. In practice, however, transition arrays often required intermediate coupling where the eigenstates are a mixture of the pure 
representation. This effect has been termed limited CI since it may be computed by including interactions between all relativistic configurations belonging to the same non-relativistic configuration [6]. A successful description of this effect should describe the progression from a single LS-UTA to a more complicated transition spectrum and finally the pure SOSA results. Models have been developed to account for this phenomenon, however, they rely on ad hoc approximations and are difficult to improve systematically $[6,7,8]$.

Recently an extension of the UTA approach was developed to partially resolve the single LSUTA feature with a number of Gaussians by exploiting properties of the moments $[9,10,11]$. First, the energy shift from the configuration average energy difference depends only on the optically active shells and is independent of the spin-orbit interaction. Secondly, the contributions to the variance from spectator subshells are independent. Finally, the limited CI effects are completely described by computing a DLA transition array including only the two optically active subshells in intermediate coupling and dressing each line with a variance provided by the spectator subshells. The partially resolved transition array (PRTA) concept conserves the known array properties, provides improved higher moments, and accounts for initial level populations while remaining computationally efficient. Systematic refinement of the spectrum is accomplished by including more subshells in the DLA calculation (albeit at greater computational cost).

The example in Fig. 1 illustrates the limited CI effects as well as the PRTA method. The full DLA calculation with intermediate coupling uses a Voigt profile with Doppler broadening plus an artificial Lorentz width of $0.01 \mathrm{eV}$. The plot includes the LS-UTA single Gaussian [2] and the sub-arrays from the SOSA approach [3]. Clearly, both statistical methods fail to describe the full DLA calculation. For comparison the PRTA with only the two active subshells included in the small DLA calculation captures the location and relative strength of the sub-arrays [10].

The purpose here is to extend the PRTA ideas to the STA method and correct inadequacies of the STA description. It has the advantages of being conceptually simple, completely describes limited CI effects, and furthermore can be systematically refined to the full detailed configuration accounting (DCA) or detailed line accounting limit. Thus, the formalism forms a framework for a hybrid scheme to combine detailed line accounting and statistical methods.

The work briefly reviews the STA method in Section 2, which also serves to introduce the notation. Section 3 describes the basic steps involved in applying PRTA approaches to 
relativistic Super Transition Arrays. Numerical examples are provided in Section 4 with conclusions offered in the final Section 5.

\section{STA method}

A super configuration $\Xi$ is designated as a set of super shells $\sigma$ with electron occupation $Q_{\sigma}$ $[4,5]$,

$$
\Xi=\prod_{\sigma \in \Xi} \sigma^{Q_{\sigma}}
$$

where a super shell consists of a group of ordinary atomic subshells $n \ell j$. The set of relativistic configurations comprising the super configuration is constructed by distributing $Q_{\sigma}$ electrons in all possible ways among the ordinary subshells subject to particle conservation,

$$
\sigma^{Q_{\sigma}}=\sum_{\left\{\sum_{\alpha \in \sigma} q_{\alpha}=Q_{\sigma}\right\}} \prod_{\alpha \in \sigma}\left(n_{\alpha} \ell_{\alpha} j\right)^{q_{\alpha}}
$$

with $q_{\alpha}$ the occupation of the $\alpha$ subshell. A one-electron radiative transition from a subshell $\alpha$ in an ordinary configuration to a subshell $\beta$ results in a second ordinary configuration and defines a transition array. The collection of such arrays defines the super transition array (STA).

The STA method makes several essential approximations [4,5]; the most relevant to the present discussion are recalled. Following the UTA description the STA method assumes that the Boltzmann factor for the level populations does not vary significantly within an ordinary configuration. On the other hand, Boltzmann factors are included from one ordinary configuration to another within a super configuration, but using a non-interacting approximation where the ordinary configuration energies are given by the sum of independent particle energies. It is also important to note that each ordinary configuration within the super configuration has a common correction to the Boltzmann factor beyond the independent electron approximation to the energy. This correction is based on Jensen's inequality for partition functions and is essential for obtaining realistic ion stage distributions [12]. Another approximation assumes the radial integrals for all ordinary configurations in the STA are obtained from the same self-consistent solution of the central potential. Finally, although the oscillator strengths depend on the transition energy, the STA method computes all with the same energy given by the STA average energy. 
The average energy of a dipole transition connecting ordinary subshells $\alpha$ and $\beta$ for the initial ordinary configuration $\tilde{C}$ can be written in the form $[2,3]$

$$
E_{C}^{\alpha \beta}=\tilde{D}_{C}^{\alpha \beta}+\sum_{\gamma \in C}\left(q_{\gamma}-\delta_{\alpha \gamma}\right) \tilde{D}_{\gamma}^{\alpha \beta}
$$

where $q_{\gamma}$ is the occupation of spin-orbital $\gamma$ in configuration $\tilde{C}$ (adopted Greek indices and tilde for the relativistic subshells and configurations respectively), $\delta_{\alpha \gamma}$ is the Kronecker delta, and the $\tilde{D}_{\gamma}^{\alpha \beta}$ are independent of the occupation numbers and include the shift of the UTA centroid from the configuration average transition energy. The centroid of the STA is the average over all ordinary configurations within a super configuration $\Xi[4,5]$,

$$
E_{\Xi}^{\alpha \beta}=\left\langle E_{C}^{\alpha \beta}\right\rangle_{\Xi}=\frac{\sum_{C \in \Xi} W_{C}^{\alpha \beta} E_{C}^{\alpha \beta}}{\sum_{C \in \Xi} W_{C}^{\alpha \beta}}
$$

Note that the configuration weighting accounts for the dipole transition strength,

$$
W_{C}^{\alpha \beta}=\omega_{\Xi}^{\alpha \beta} q_{\alpha}\left(g_{\beta}-q_{\beta}\right) P_{C}
$$

as well as the Boltzmann configuration probability

$$
P_{C}=\frac{1}{\Gamma} G_{C} e^{-\left(E_{C}-\mu N_{C}\right) / T}=\left\{\frac{1}{\Gamma} e^{-\Delta E_{\Xi} / T}\right\} \prod_{\gamma \in C} \frac{g_{\gamma} !}{q_{\gamma} !\left(g_{\gamma}-q_{\gamma}\right) !} X_{\gamma}^{q_{\gamma}}
$$

where $T$ is the plasma temperature in energy units, $\mu$ is the chemical potential, and $\Delta E_{\Xi}$ is a correction to the Boltzmann factor beyond the independent electron approximation [12]. The independent subshell Boltzmann factor is

$$
X_{\gamma}=e^{-\left(\varepsilon_{\gamma}-\mu\right) / T}
$$

with $\varepsilon_{\gamma}$ and $g_{\gamma}$ the removal energy and the degeneracy for subshell $\gamma$, respectively, and the grand partition function

$$
\Gamma=\sum_{\Xi} P_{\Xi}=\sum_{\Xi}\left\{\sum_{C \in \Xi} P_{C}\right\}
$$


Note well that the absorption transition probability from subshell $\alpha$ to subshell $\beta$ is approximated using the super configuration average energy making it independent of the ordinary configurations,

$$
\omega_{\Xi}^{\alpha \beta}=\frac{\pi e^{2} h}{m c} \frac{E_{\Xi}^{\alpha \beta} S^{\alpha \beta}}{3}
$$

with

$$
S^{\alpha \beta} \propto\left(\begin{array}{ccc}
j_{\alpha} & 1 & j_{\beta} \\
1 / 2 & 0 & -1 / 2
\end{array}\right)^{2}=\frac{1}{4}\left(\begin{array}{ccc}
\ell_{\alpha} & 1 & \ell_{\beta} \\
0 & 0 & 0
\end{array}\right)^{2} g_{\alpha} g_{\beta}\left\{\begin{array}{ccc}
j_{\alpha} & 1 & j_{\beta} \\
\ell_{\beta} & 1 / 2 & \ell_{\alpha}
\end{array}\right\}^{2}
$$

the dipole line strength connecting subshells $\alpha$ and $\beta$ for a hypothetical one-electron atom $[13,14]$.

The expression for the STA centroid simplifies to a form

$$
E_{\Xi}^{\alpha \beta}=\tilde{D}_{0}^{\alpha \beta}+\sum_{\sigma \in \Xi} \varepsilon_{\sigma}^{\alpha \beta}\left(Q_{\sigma}-\delta_{\sigma, \sigma_{\alpha}}\right)
$$

which is linearly additive in the independent super shell quantities

$$
\varepsilon_{\sigma}^{\alpha \beta}(Q)=\sum_{\gamma \in \sigma} g_{\gamma}^{\alpha \beta} X_{\gamma} \tilde{D}_{\gamma}^{\alpha \beta} \frac{U^{\sigma}\left(Q-\delta_{\sigma, \sigma_{\gamma}} \mid \vec{g}^{\alpha \beta \gamma}\right)}{U^{\sigma}\left(Q \mid \vec{g}^{\alpha \beta}\right)}
$$

where $\sigma_{\alpha}$ identifies the super shell containing the subshell $\alpha$ and super shell partition functions are explicitly defined as

$$
U^{\sigma}(Q \mid \vec{g})=\sum_{\left\{\sum_{\gamma \in \sigma} q_{\gamma}=Q\right.} \prod_{\gamma \in \sigma} \frac{g_{\gamma} !}{q_{\gamma} !\left(g_{\gamma}-q_{\gamma}\right) !} X_{\gamma}^{q_{\gamma}}
$$

with

$$
\vec{g}^{\alpha \beta \gamma \cdots}=\left\{g_{v}^{\alpha \beta \gamma \cdots}\right\} \equiv\left\{g_{v}-\delta_{\alpha v}-\delta_{\beta v}-\delta_{w} \cdots\right\}
$$

a modified vector of orbital degeneracies. Careful inspection reveals that the centroid depends only on the two subshells of the optically active electrons.

Under the same assumptions and after lengthy manipulations, the variance of the STA can be written in the form $[4,5]$ 


$$
\Delta_{\Xi}^{\alpha \beta}=\sum_{\sigma \in \Xi} \Delta_{\sigma}^{\alpha \beta}
$$

where $\Delta_{\sigma}^{\alpha \beta}$ contains the variance contribution from the individual configuration average energies including energy shifts due to the selection rules (the UTA shifts) and the energy level splitting within the ordinary configurations (the UTA widths). An important feature of $\Delta_{\Xi}^{\alpha \beta}$ is that the contributions from the super shells add independently.

The STA strength for photon absorption by a dipole transition from subshell $\alpha$ to subshell $\beta$ is given by $[4,5]$

$$
\begin{aligned}
I_{\Xi}^{\alpha \beta} & =\sum_{C \in \Xi} W_{C}^{\alpha \beta}=\left\{\frac{1}{\Gamma} e^{-\Delta E_{\Xi} / T}\right\} \omega_{\Xi}^{\alpha \beta} X_{\alpha} g_{\alpha} g_{\beta} \prod_{\sigma \in \Xi} U^{\sigma}\left(Q_{\sigma}-\delta_{\sigma, \sigma_{\alpha}} \mid \vec{g}^{\alpha \beta}\right) \\
& =P_{\Xi} \omega_{\Xi}^{\alpha \beta} X_{\alpha} g_{\alpha} g_{\beta} \prod_{\sigma \in \Xi} \frac{U^{\sigma}\left(Q_{\sigma}-\delta_{\sigma, \sigma_{\alpha}} \mid \vec{g}^{\alpha \beta}\right)}{U^{\sigma}\left(Q_{\sigma} \mid \vec{g}\right)}
\end{aligned}
$$

where $P_{\Xi}$ is the occupation probability of the super configuration $\Xi$.

Finally, the transition array is approximated by a Gaussian and the STA spectrum is given by

$$
L_{\Xi}^{\alpha \beta}(v)=I_{\Xi}^{\alpha \beta} G\left(h v ; E_{\Xi}^{\alpha \beta}, \Delta_{\Xi}^{\alpha \beta}\right)
$$

with

$$
G(h v ; E, \Delta)=\frac{1}{\sqrt{2 \pi \Delta}} \exp \left\{-\frac{(h v-E)^{2}}{2 \Delta}\right\}
$$

For clarity the intrinsic line profile was neglected, but can be included by convolving it with the normalized Gaussian in Eq. (2.15).

\section{Super-PRTA}

As mentioned above, the PRTA ideas are extended to the STA method. The specific application describes the limited CI effects with a DLA in intermediate coupling. More generally, the extended concept permits stepwise refinement of the super transition array method towards the line-by-line limit in ordinary configurations. Thus, the formalism forms a framework for a hybrid scheme to combine detailed line accounting and statistical methods.

\subsection{Reduction from relativistic to non-relativistic STA}


Analogous to the PRTA development of ordinary configurations, the generalization to super configurations proceeds not from moment formula generated in a JJ basis with relativistic spinorbitals as described in Section 2, but from intermediate coupling calculations utilizing a nonrelativistic LS basis. A non-relativistic super configuration $\Omega$ is a set of super shells $S$, which now consists of a group of ordinary non-relativistic orbitals $n \ell$ with electron occupation $Q_{s}$. A non-relativistic orbital label $b$ (Latin alphabet for non-relativistic orbitals $n \ell$ ) can be associated with up to two relativistic spin orbitals $\beta$. Henceforth relativistic configurations, $\tilde{C}$, are denoted with the tilde to distinguish them from non-relativistic configurations, $C$.

By convention relativistic super shell structure assigns the spin up and spin down orbitals to the same super shell so that each relativistic super configuration

$$
\begin{array}{r}
\Xi \equiv\left[1 s_{1 / 2} 2 s_{1 / 2} 2 p_{1 / 2} 2 p_{3 / 2}\right]^{10}\left(3 s_{1 / 2} 3 p_{1 / 2} 3 p_{3 / 2} 3 d_{3 / 2} 3 d_{5 / 2}\right)^{17} \\
\left(4 s_{1 / 2} 4 p_{1 / 2} 4 p_{3 / 2} 4 d_{3 / 2} 4 d_{5 / 2} 4 f_{5 / 2} 4 f_{7 / 2}\right)^{2}\left(5 s_{1 / 2} \cdots\right)^{1}
\end{array}
$$

is associated with the non-relativistic super configuration

$$
\Omega \equiv[1 s 2 s 2 p]^{10}(3 s 3 p 3 d)^{17}(4 s 4 p 4 d 4 f)^{2}(5 s 5 p \ldots)^{1}
$$

with the same probability. The three possible relativistic super transition arrays are subsumed by a single non-relativistic transition array. As an example, consider

$$
\left.\begin{array}{l}
3 d_{3 / 2} \rightarrow 4 f_{5 / 2} \\
3 d_{5 / 2} \rightarrow 4 f_{5 / 2} \\
3 d_{5 / 2} \rightarrow 4 f_{7 / 2}
\end{array}\right\} \Rightarrow 3 d \rightarrow 4 f
$$

whose energy centroid is given by

$$
E_{\Omega}^{a b}=\frac{\sum_{C \in \Omega} W_{C}^{a b} E_{C}^{a b}}{\sum_{C \in \Omega} W_{C}^{a b}}
$$

with the average energy of a non-relativistic configuration (including LS-UTA shifts)

$$
E_{C}^{a b} \equiv D_{0}^{a b}+\sum_{i \in C}\left(q_{i}-\delta_{i a}\right) D_{i}^{a b}
$$

Note that the non-relativistic orbital parameters $D_{i}^{a b}$ can be written as spin averages over relativistic orbital parameters $\tilde{D}_{\gamma}^{\alpha \beta}$. 
The configuration weighting, defined exactly as

$$
W_{C}^{a b} \equiv \sum_{\alpha \in a} \sum_{\beta \in b}\left\{\sum_{\tilde{C} \in C} W_{\tilde{C}}^{\alpha \beta}\right\}
$$

can be written in a form analogous to the relativistic case by making two weak approximations within the relativistic configuration Boltzmann factor. Specifically,

$$
E_{\tilde{C}} \cong E_{C} \quad \text { for all } \tilde{C} \in C
$$

and that the already approximate average energy in the transition probability be replaced by

$$
E_{\Xi}^{\alpha \beta} \cong E_{\Omega}^{a b}
$$

Then

$$
\begin{aligned}
& W_{c}^{a b}=\omega_{\Omega}^{a b} q_{a}\left(g_{b}-q_{b}\right) P_{C} \\
& P_{C} \equiv \frac{1}{\Gamma} G_{C} e^{-\beta\left(E_{c}-\mu N_{c}\right)} \\
& \omega_{\Omega}^{a b}=\frac{\pi e^{2} h}{m c} \frac{E_{\Omega}^{a b} S^{a b}}{3}
\end{aligned}
$$

where (see Appendix B of [6])

$$
S^{a b} \equiv\left(\frac{1}{g_{a} g_{b}}\right) \sum_{\alpha \in a} \sum_{\beta \in b}\left\{g_{\alpha} g_{\beta} S^{\alpha \beta}\right\} \approx \frac{1}{2}\left(\begin{array}{ccc}
l_{a} & 1 & l_{b} \\
0 & 0 & 0
\end{array}\right)^{2}
$$

With the judicious choice in Eq. (3.1.8), the strength of the transition array

$$
I_{\Omega}^{a b}=\sum_{C \in \Omega} W_{c}^{a b}
$$

conserves the sum of JTA's (details are relegated to Appendix A). It is emphasized that both the centroid and variance are independent of the choice of this parameter (it cancels in the defining numerator and normalizing denominator) and its value is constrained only in the asymptotic DCA limit of super shell refinement. Note also that the normalizing factor of the grand partition function remains that of the relativistic accounting.

The variance can be written in the form

$$
\Delta_{\Omega}^{a b}=\Delta_{\text {spin-orb }}^{a b}+\sum_{s \in \Omega} \Delta_{s}^{a b}
$$

The electrostatic contribution to the variance add linearly as independent super shell quantities and depends on non-relativistic Slater integral parameters that are evaluated as spin averages 
over their relativistic counterparts [15]. The effects of intermediate coupling depends only on the two active subshells independent of their occupations and contributes only to the variance as

$$
\Delta_{\text {spin-orb }}^{a b} \equiv\left\{\frac{1}{4}\left(\xi_{b}-\xi_{a}\right)\left[\xi_{b} l_{b}\left(l_{b}+1\right)-\xi_{a} l_{a}\left(l_{a}+1\right)\right]+\frac{1}{2} \xi_{b} \xi_{a}\right\}
$$

where $\xi_{a}$ stands for the spin-orbit radial integral of the $a$ orbital [16]. The shift from configuration average energy depends only on the two active shells.

\subsection{DLA calculations for active subshells}

The present formulation now follows closely those developed in the PRTA method for ordinary configurations in intermediate coupling [10]. The plan is first to resolve a given a super configuration $\Omega$ into a set of daughter super configurations $\{\Phi\}$ such that each daughter has each active subshell isolated in its own super shell. This results in a modest multiplicity in the number of daughter super configurations that is processed to produce a spectrum. It is emphasized that the present description must use the LS representation together with spin-orbit parameters to perform the necessary intermediate angular momentum coupling.

To proceed, consider the STA for the $a=3 d$ to $b=4 f$ transitions with initial super configuration,

$$
\Omega=(1 s 2 s 2 p)^{10}(3 s 3 p 3 d)^{17}(4 s 4 p 4 d 4 f)^{2}
$$

The set of daughter super configurations is then given by

$$
\begin{array}{ll}
(1 s 2 s 2 p)^{10}(3 s 3 p)^{8} 3 d^{9}(4 s 4 p 4 d)^{2} & (1 s 2 s 2 p)^{10}(3 s 3 p)^{7} 3 d^{10}(4 s 4 p 4 d)^{2} \\
(1 s 2 s 2 p)^{10}(3 s 3 p)^{8} 3 d^{9}(4 s 4 p 4 d)^{1} 4 f & (1 s 2 s 2 p)^{10}(3 s 3 p)^{7} 3 d^{10}(4 s 4 p 4 d)^{1} 4 f \\
(1 s 2 s 2 p)^{10}(3 s 3 p)^{8} 3 d^{9} 4 f^{2} & (1 s 2 s 2 p)^{10}(3 s 3 p)^{7} 3 d^{10} 4 f^{2}
\end{array}
$$

where each daughter super configuration has explicitly isolated the optically active subshells.

At this juncture the Super-PRTA algorithm admits some ambiguities. The STA model assumes that all the UTA oscillator strengths within the STA are computed with identical energies typically taken as the centroid of the array. Thus

$$
\begin{aligned}
& I_{\Omega}^{a b} \approx E_{\Omega}^{a b} \sum_{C \in \Omega} q_{a}\left(g_{b}-q_{b}\right) P_{C}^{\Omega} \\
& I_{\Phi}^{a b} \approx E_{\Phi}^{a b} \sum_{C \in \Phi} q_{a}\left(g_{b}-q_{b}\right) P_{C}^{\Phi}
\end{aligned}
$$


and while the specialization of $E_{\Phi}^{a b}$ (as well as a specialization of the correction $\Delta E_{\Phi}$ to the probability distribution) is in principle more accurate for $I_{\Phi}^{a b}$, as a consequence strength is not strictly conserved:

$$
\sum_{\Phi \in \Omega} I_{\Phi}^{a b} \neq I_{\Omega}^{a b}
$$

In the present calculations the cruder approximation was used whereas the configuration weighting for all daughter super configurations is inherited from the parent super configuration (i.e., calculated using the formula for the parent); thus, conserving strength.

The method next performs a DLA calculation including only the two active subshells. For the aforementioned example the 6 transition arrays

$$
\begin{array}{rlrl}
3 d^{9} & \rightarrow 3 d^{8} 4 f & 3 d^{10} & \rightarrow 3 d^{9} 4 f \\
3 d^{9} 4 f & \rightarrow 3 d^{8} 4 f^{2} & 3 d^{10} 4 f & \rightarrow 3 d^{9} 4 f^{2} \\
3 d^{9} 4 f^{2} & \rightarrow 3 d^{8} 4 f^{3} & 3 d^{10} 4 f^{2} & \rightarrow 3 d^{9} 4 f^{3}
\end{array}
$$

are required. It should be mentioned that DLA calculations with open shells of equivalent $f$ and $g$ shell electrons of non-trivial occupancy are accessed with surprisingly frequency, so Racah based DLA atomic structure packages [16] may require extended tables of coefficients of fractional parentage $[17,18,19]$. For such DLA transitions, alternative atomic structure packages, such as those employing determinant bases, may trade speed for simplicity and reduced memory requirements [20,21].

Similar to the PRTA approach [10], each line from the DLA calculations is dressed by the contribution from the spectator super shells. Thus, the daughter STA spectrum is given by

$$
L_{\Phi}^{a b}(h v)=J_{\Phi}^{a b} \sum_{j=1}^{N_{\Phi}^{a b}} g_{j} e^{-u_{j} / k T} S_{j} G\left(h v ; h v_{j}, \hat{\Delta}_{\Phi}^{a b}\right)
$$

where the DLA calculation yields $N_{\Phi}^{a b}$ lines with $h v_{j}, s_{j}, g_{j}$, and $u_{j}$ the line energy, line strength, initial level degeneracy, and initial level energy for the $\mathrm{j}^{\text {th }}$ DLA transition, respectively. The probabilities and line strengths from the DLA calculation are normalized so that

$$
I_{\Phi}^{a b}=J_{\Phi}^{a b}\left\{\sum_{j=1}^{N_{\Phi}^{a b}} g_{j} e^{-u_{j} / T}\right\}\left\{\sum_{j=1}^{N_{\Phi}^{a b}} s_{j}\right\}
$$


defines $J_{\Phi}^{a b}$ with $I_{\Phi}^{a b}$ given in Eq. (3.2.3). The variance accounts for the contribution of the spectator electrons to the width of the feature

$$
\hat{\Delta}_{\Phi}^{a b}=\sum_{\substack{s \in \Phi \\ s \neq s_{a}, s_{b}}} \Delta_{s}^{a b}
$$

and excludes the contribution from the optically active super shells. The expression in Eq. (3.2.6) accounts for the Boltzmann distribution of the initial levels in the DLA calculation. This can impact the spectrum if the configuration term structure (including the spin-orbit splitting) in the initial optically active subshell is comparable to the plasma temperature. In the sample calculations provided here this effect has been omitted.

The spectrum replacing the parent STA results, $L_{\Omega}^{a b}(v)$, is the weighted sum of all the STA daughters,

$$
L_{\Omega}^{a b}(v) \rightarrow \sum_{\Phi \in \Omega} L_{\Phi}^{a b}(v)
$$

Note that the weighted strength $I_{\Phi}^{a b}$ appears in the expression for $L_{\Phi}^{a b}$ and already includes the relative probability of the daughter STA.

\section{Numerical examples}

The method described in Section 3 is illustrated with two examples. The first focuses on a single super transition array. Since the line spectrum has temperature dependence not only from Doppler broadening but also from the Boltzmann weighting of the initial levels and the spectator electron variance, the temperature in the first example is chosen so that the featured ion charge state agrees with the ionization average from a Thomas-Fermi [22] calculation at one-hundredth normal density of the material. Thus, the example is representative of conditions relevant to experiments and laboratory applications. It involves the $3 d$ to $4 f$ transitions in Section 3.2 for $\mathrm{Er}^{39+}$ at $\rho=0.09 \mathrm{~g} / \mathrm{cm}^{3}$ and $T=295 \mathrm{eV}$. The line spectrum for the Super PRTA approximation is displayed in Fig. 2. Also in the figure is the total PRTA line spectrum including only active subshells in the small DLA for all 30 ordinary configurations subsumed in the super configuration. These are contrasted with the spectra from the SOSA approach generated with DCA or STA treatments. A Voigt profile describes the intrinsic line shape where the Gaussian contribution is due to Doppler broadening and a Lorentz component due to electron impact [23] 
plus natural width. For the parent STA the Gaussian width includes the total STA variance where the daughters only include the variance from the spectator super shells. Since even two open shell configurations can generate myriad lines in intermediate coupling [24] and a common line profile is assumed for each member of the transition array, an efficient method for placing line profiles on an energy mesh can be used to advantage [25].

A second example is a complete opacity calculation for Fe at $\mathrm{T}=20 \mathrm{eV}$ and $\rho=10^{-4} \mathrm{~g} / \mathrm{cm}^{3}$ relevant to astrophysical applications [26]. These conditions, besides highlighting the sensitivity of the Rosseland mean to array porosity exhibited by DLA, also served as a motivation to include intermediate coupling effects [6,7]. As seen in Fig.3 the limited CI effect is manifested by shifting the strengths of the $n=3$ to 3 transition arrays in the $80-100 \mathrm{eV}$ range, as evidenced by the TOPAZ intermediate coupling DLA calculations [27] absent in SOSA treatments either with TOPAZ DCA or VISTA [28] super configuration accounting. The latter are displayed in Fig.4.

The important limited CI effects are captured by the Super-PRTA algorithm as shown in Fig. 5, which has the ancillary effect of introducing some porosity into the $\Delta n=0$ transitions. Note that the net Super PRTA effect on $\Delta n \neq 0$ transition arrays, while still dressing detailed lines with spectator variances, is to leave essentially unresolved arrays. As illustrated by Fig. 6, the Super-PRTA captures the main effects of intermediate coupling, but within a superunresolved transition array approximation. In situations where array porosity is an important factor in the determination of Rosseland means, further refinement of the transition array accounting is still required.

\section{Conclusions}

A method to resolve partially the super transition array (STA) into daughter STA's that accurately describes the evolution from pure LS conditions to the spin-orbit split sub-arrays was presented. The method uses a relatively small detailed line accounting (DLA) calculation with intermediate angular momentum coupling for the optically active subshells. The approach replaces ad hoc schemes to account for configuration interactions between relativistic subconfigurations while remaining computationally efficient.

Although the present work emphasized limited configuration interaction effects captured by treating only the active subshells by a DLA method, the formalism permits a stepwise refinement of a super transition array to the limit of DLA in ordinary configurations. It is possible to 
envision including additional open subshells in the small DLA calculation, further refining the super shell structure, or both. Thus, the proposed method can be generalized to construct a hybrid super-PRTA description combining DLA with statistical methods. A step in that direction has been taken in order to account for highly excited spectator electrons with statistical methods in line-by-line opacity calculations [29].

\section{Appendix A. Conservation of line strength}

In this appendix the approximate conservation of the strength of a non-relativistic transition array with the sum of the strengths of relativistic JTA's comprising the array is derived. This is demonstrated by neglecting the energy differences between the relativistic spin up and down orbitals, whence the neglect of the Boltzmann factors in the evaluation of Eq. (3.1.6). Conserving the sum of JTA's then reduces to establishing the identity

$$
\frac{\sum_{\tilde{C} \in C} G_{\tilde{C}} \sum_{\alpha \in a} \sum_{\beta \in b} q_{\alpha}\left(g_{\beta}-q_{\beta}\right) S^{\alpha \beta}}{\sum_{\tilde{C} \in C} G_{\tilde{C}}}=q_{a}\left(g_{b}-q_{b}\right) S^{a b}
$$

where the sum is over all relativistic configurations $\tilde{C}$ contained in the nonrelativistic configuration $C$. This is demonstrated by using the binomial identities [Eq. (9.92) in Ref. 16]

$$
\begin{gathered}
\sum_{q_{\alpha}=0}^{g_{a}} \sum_{\substack{q_{\alpha^{\prime}}=0 \\
q_{\alpha}+q_{\alpha^{\prime}}=q_{a}}}^{g_{a}}\left(\begin{array}{c}
g_{\alpha} \\
q_{\alpha}
\end{array}\right)\left(\begin{array}{c}
g_{\alpha^{\prime}} \\
q_{\alpha^{\prime}}
\end{array}\right)=\left(\begin{array}{c}
g_{\alpha}+g_{\alpha^{\prime}} \\
q_{a}
\end{array}\right)=\left(\begin{array}{c}
g_{a} \\
q_{a}
\end{array}\right) \\
\sum_{q_{\alpha}=0}^{g_{a}} \sum_{\substack{q_{\alpha^{\prime}}=0 \\
q_{\alpha}+q_{\alpha^{\prime}}=q_{a}}}^{g_{a}}\left\{q_{\alpha}\right\}\left(\begin{array}{c}
g_{\alpha} \\
q_{\alpha}
\end{array}\right)\left(\begin{array}{c}
g_{\alpha^{\prime}} \\
q_{\alpha^{\prime}}
\end{array}\right)=\left\{\frac{q_{a}}{g_{a}}\right\}\left\{g_{\alpha}\right\}\left(\begin{array}{c}
g_{a} \\
q_{a}
\end{array}\right)
\end{gathered}
$$

along with the definition Eq. (3.1.9d)

Acknowledgments: This work performed under the auspices of the U.S. Department of Energy by Lawrence Livermore National Laboratory under Contract DE-AC52-07NA27344. 


\section{References}

[1] Q. Porcherot, J.-Ch. Pain, F. Gilleron, T. Blenski, HEDP 7, 234(2011)

[2] C. Bauche-Arnoult, J. Bauche \& M. Klapisch, Phys.Rev. A20, 2424(1979)

[3] C. Bauche-Arnoult, J. Bauche \& M. Klapisch, Phys.Rev. A31, 2248(1985)

[4] A. Bar-Shalom et al., Phys.Rev. A40, 3183(1989)

[5] A. Bar-Shalom et al., Phys.Rev. E51, 4882(1995)

[6] A. Bar-Shalom, J. Oreg, \& W. H. Goldstein, JQSRT 51, 27(1994)

[7] A. Bar-Shalom, J. Oreg, M. Klapisch \& T. Lehecka, Phys.Rev. E59, 3512(1999)

[8] F. Gilleron, J. Bauche \& C. Bauche-Arnoult, J.Phys. B40, 3057(2007)

[9] C.A. Iglesias \& V. Sonnad, HEDP 8, 154(2012)

[10] C.A. Iglesias, HEDP 8, 253(2012)

[11] C.A. Iglesias, HEDP 8, 260(2012)

[12] G. Faussurier, Phys. Rev. E 59, 7096(1999)

[13] I. P. Grant, "Relativistic Calculation of Atomic Structure", p.747 in "Advances in Physics" (1970). See Eq. (5.14)

[14] I. P. Grant, J. Phys. B 7, 1458(1974)

[15] F. P. Larkins, J. Phys. B 9, 37(1976)

[16] R. Cowan, The Theory of Atomic Structure (California Press, Berkeley, 1974)

[17] D. Allison and J. McNulty, Comp. Phys. Commun. 8, 246(1974)

[18] B. Judd, Phys. Rev. 173, 40(1968)

[19] B. Judd \& S. Li, J. Phys. B: At. Mol. Opt. Phys. 22, 2057(1989)

[20] E. Hill, JQSRT 140, 1(2014)

[21] E. Hill, JQSRT 147, 71(2014)

[22] R.P. Feynman, N Metropolis \& E. Teller, Phys.Rev. 75, 1561(1949)

[23] M.S. Dimitrijevic \& N. Konjevic, JQSRT 24, 451(1980)

[24] J. Bauche \& C. Bauche-Arnoult, Comp. Phys. Rep. 12, 1(1990)

[25] V. Sonnad \& C. A. Iglesias, HEDP 7, 42(2011)

[26] F.J. Rogers \& C.A. Iglesias, Science 263, 50(1994)

[27] C.A. Iglesias, M.H. Chen, V. Sonnad et al., JQSRT 81, 227(2003) 
[28] VISTA is a relativistic LTE opacity code developed at Lawrence Livermore National Laboratory based on STA theory using Dirac-Hartree-Slater methods with Breit and QED corrections for the atomic data.

[29] J.-Ch. Pain \& F. Gilleron, HEDP (submitted to HEDP) 


\section{Figure Captions}

Figure 1. The transition array $[\mathrm{Mg}] 3 \mathrm{p}^{5} 3 \mathrm{~d}^{3} 4 \mathrm{~s} 4 \mathrm{p}$ to $[\mathrm{Mg}] 3 \mathrm{p}^{4} 3 \mathrm{~d}^{4} 4 \mathrm{~s} 4 \mathrm{p}$ for $\mathrm{In}^{27+}$ at $T=190 \mathrm{eV}$ : full DLA intermediate coupling calculation (grey); LS-UTA (dash); SOSA (dot-dash); and PRTA (solid).

Figure 2. The $\mathrm{Er}^{39+} 3 \mathrm{~d}$ to $4 \mathrm{~d}$ transition arrays with initial super configuration $[\mathrm{Ne}][\mathrm{M}]^{17}[\mathrm{~N}]^{2}$ at $\mathrm{T}=295 \mathrm{eV}$ and $\rho=0.09 \mathrm{~g} / \mathrm{cm}^{3}$ : STA parent (dash); 30 ordinary configurations with DCASOSA (dot-dash) and DLA-PRTA (grey); and Super-PRTA for the daughters (solid).

Figure 3. Frequency dependent opacity as a function of photon energy for Fe plasma at $\mathrm{T}=20 \mathrm{eV}$ and $\rho=10^{-4} \mathrm{~g} / \mathrm{cm}^{3}$ : TOPAZ SOSA (black) and TOPAZ DLA with intermediate coupling (grey). The Rosseland mean opacity increased by 11 percent $\left(6006 \mathrm{~cm}^{2} / \mathrm{g}\right.$ for SOSA to 6673 $\mathrm{cm}^{2} / \mathrm{g}$ for DLA).

Figure 4. Same as Fig. 3: TOPAZ DCA-SOSA as in Fig. 3 (thin black) and STA-SOSA (thick grey). Although both approaches give nearly identical ionization balance $\left(Z^{*}=8.70\right.$ with DCA and 8.67 for STA) the Rosseland mean opacity differs significantly $\left(6670 \mathrm{~cm}^{2} / \mathrm{g}\right.$ with DCA and $7705 \mathrm{~cm}^{2} / \mathrm{g}$ for STA).

Figure 5. Same as Fig. 3: STA-SOSA as in Fig. 3 (grey) and Super-PRTA demonstrating intermediate coupling effects (thin-black). The Rosseland mean opacity increased by $11 \%$ from $7705 \mathrm{~cm}^{2} / \mathrm{g}$ for SOSA to $8517 \mathrm{~cm}^{2} / \mathrm{g}$ for PRTA.

Figure 6. Same as Fig. 3: Super-PRTA as in Fig.5 (thin black); TOPAZ DLA with intermediate coupling (grey). 
Figure 1

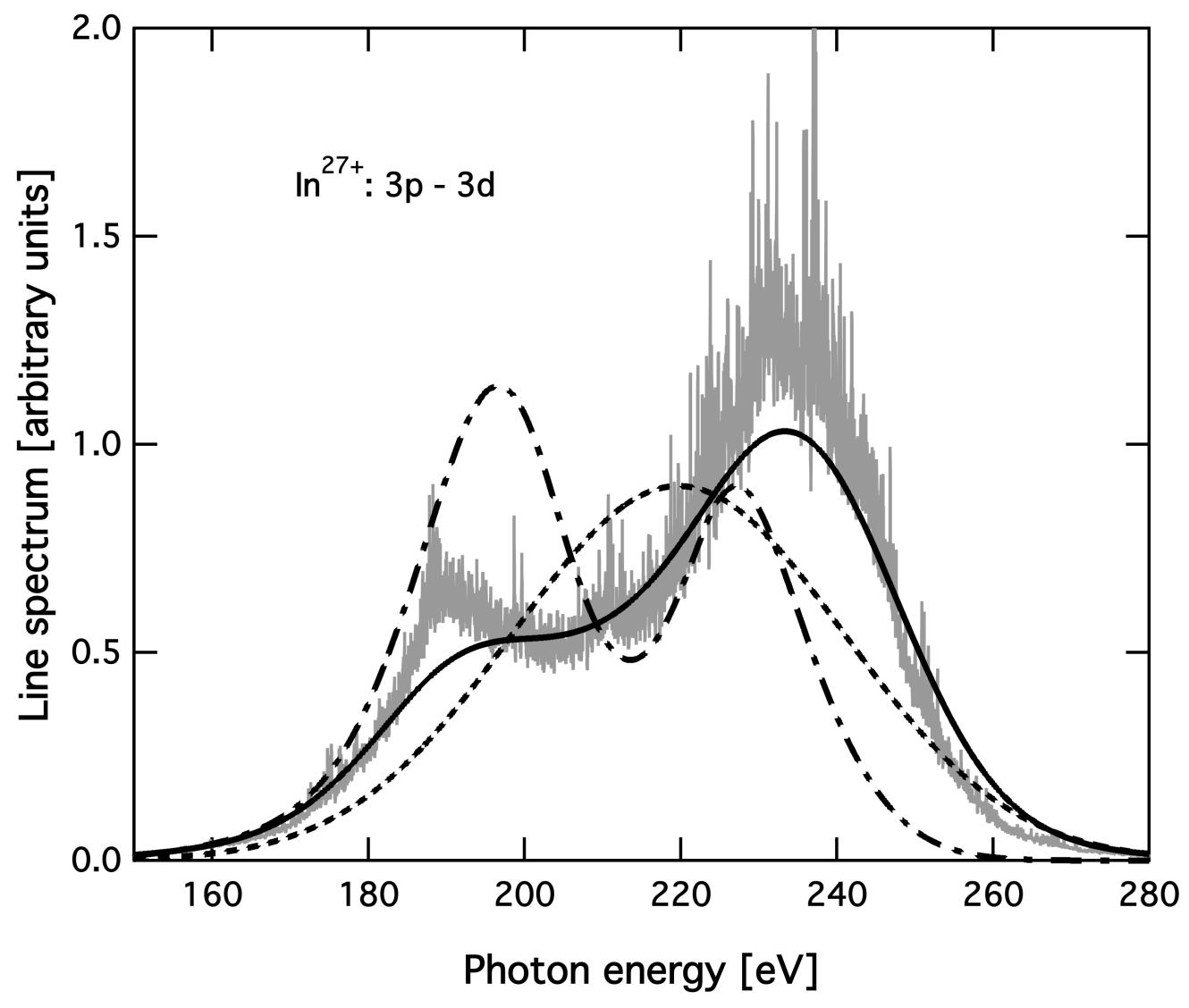




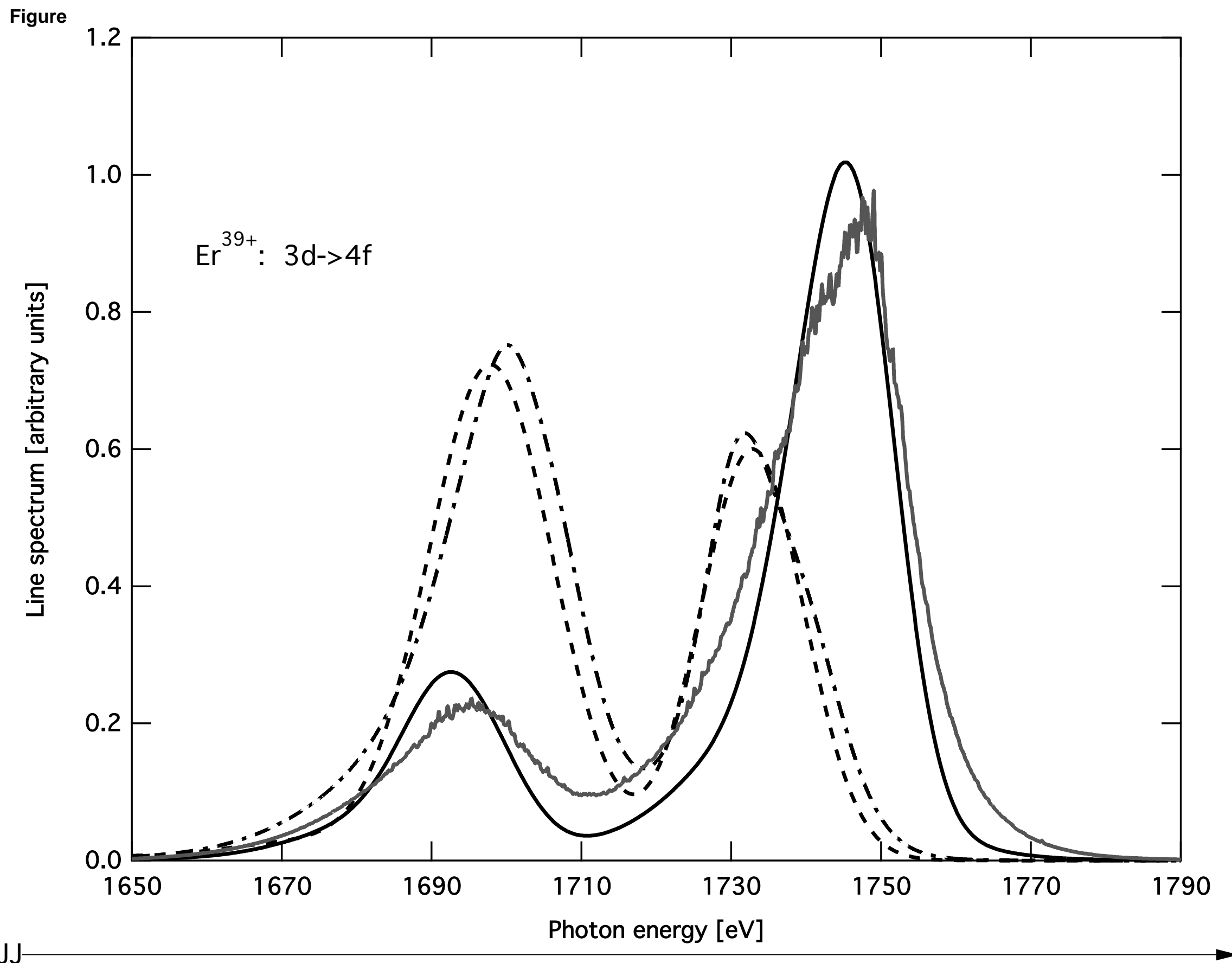




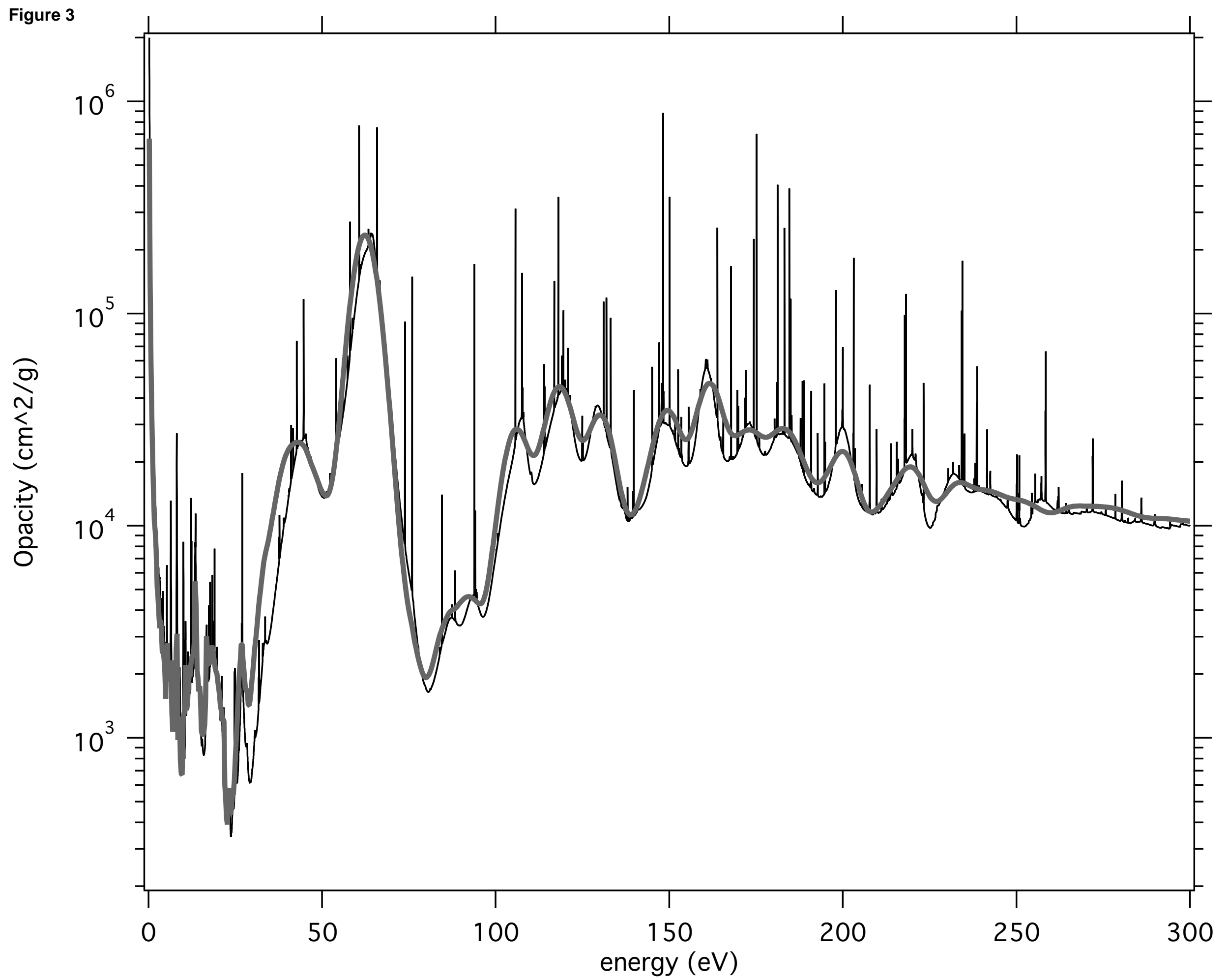


